\title{
ORIGINAL
}

\section{DIFERENCIAS EN LA ESPERANZA DE VIDA LIBRE DE DISCAPACIDAD POR SEXO Y COMUNIDADES AUTÓNOMAS EN ESPAÑA (*)}

\author{
Rosa Gispert (1), Miguel Ruíz-Ramos (2), Mª Arán Barés (1), Francisco Viciana (3) y Guillém \\ Clot-Razquin (1)
}

(1) Servei d'Informació i Estudis. Departament de Salut. Generalitat de Catalunya.

(2) Conserjería de Salud de Andalucía.

(3) Universidad de Sevilla.

\section{RESUMEN}

Fundamentos: La mejora de la salud de la población es el principal objetivo y el mayor reto del sistema sanitario y para monitorizarla se dispone de indicadores como la esperanza de vida libre de discapacidad (EVLD). El objetivo es analizar la distribución de este indicador por comunidades autónomas (CCAA) en España.

Métodos: Los datos de mortalidad, de población y de discapacidad para el año 1999 proceden del INE. La EVLD se ha calculado por el método de Sullivan, que pondera la tabla de vida en función del estado de discapacidad, por sexo y CCAA. También se ha estimado el error estándar de la EVLD, la expectativa de discapacidad y la proporción del tiempo vivido libre de discapacidad.

Resultados: En 1999 la EVLD al nacer fue de 68,5 años en hombres y de 72,2 años en mujeres. El tiempo vivido sin discapacidades fue superior en hombres $(91 \%)$ que en mujeres $(87,7 \%)$ y la expectativa de discapacidad de 6,8 años y de 10,1 años respectivamente. La diversidad entre comunidades es superior en la EVLD que en la esperanza de vida (EV). Las CCAA con mayor esperanza de vida no coinciden siempre con las que tienen una mayor proporción de tiempo vivido sin discapacidad.

Conclusiones: Una mayor EV no significa necesariamente una mejor salud, tal como se asume tradicionalmente. La EVLD es útil para evidenciar diferencias del estado de salud en la población española.

Palabras clave: Esperanza de vida. Longevidad. Años de vida ajustados por calidad.

Correspondencia a:

Rosa Gispert. Servei d'Informació i Estudis.

Departament de Salut.

C/ Travessera de les Corts 131-159. Pavelló Ave Maria 08028 Barcelona

Correo electrónico: rosa.gispert@ gencat.net

(*) El presente trabajo ha contado con ayuda parcial del

ISC III (proyecto FIS PI052650)

\section{ABSTRACT \\ Differences in Disability-Free Life Expectancy by Gender and Autonomous Regions in Spain}

Background: Improvement of population health is the main aim and an important challenge for the health system. To monitor the population health indicators like disability-free life expectancy (DFLE) have been implemented. The purpose of this paper was to analyze the geographical distribution of DFLE according to autonomous regions in Spain.

Methods: Data of mortality, population and disability for the year 1999, provided by the National Institute of Statistics (INE), were used. To calculate DFLE by gender and region we used the Sullivan method that weights the expected time to live according to the status of disablement of the population. The standard error of DFLE, the expectation of disability and the proportion of time lived free of disability have also been estimated.

Results: In 1999 the DFLE at birth in Spain was 68.5 year for men and 72.2 years in women. Men lived proportionally more time free of disability than women $(91 \%$ versus $87.7 \%)$ with an expectation of disability of 6.8 and 10.1 years respectively. Variability among regions was higher in DFLE than in life expectancy (LE). The regions with highest LE are not always those with the highest proportion of time lived without disability.

Conclusions: Highest life expectancy does not always mean best health as it has been assumed currently. The DFLE indicator is a useful tool to show health status differences among the Spanish population.

Key words: Life expectancy. Quality-adjusted life years. Longevity. 


\section{INTRODUCCIÓN}

La monitorización del estado de salud de la población desde una perspectiva de salud pública sigue sustentándose en indicadores basados fundamentalmente en datos de mortalidad. Afortunadamente, desde hace varias décadas la supervivencia y la edad media en el momento de la muerte siguen aumentando y sigue creciendo la esperanza de vida $(E V)$ de la población, aunque más moderadamente. En este contexto los indicadores de mortalidad se muestran cada vez más insuficientes para reflejar un concepto de salud acorde con objetivos del sistema sanitario, como son restaurar la salud y prevenir la enfermedad y la expectativa de la población de vivir más y mejor ${ }^{1}$.

En algo más de una década se han propuesto diversos tipos de indicadores que incorporan información complementaria a la de mortalidad, como los que miden la discapacidad, la restricción de actividad y la percepción de salud como forma de reflejar otros aspectos de la salud más ligados a la calidad de vida ${ }^{2,3}$. Las diferencias entre ellos se basan en los objetivos y la utilización para la que se proponen: evaluación económica, priorización de intervenciones sanitarias o monitorización del estado de salud. También en que utilizan fuentes de información y/o métodos de cálculo distintos ${ }^{4,5}$. Estos indicadores tienen ventajas e inconvenientes pero, puesto que incorporan datos de morbilidad, el problema común es la falta de comparabilidad debida a la diversidad de los conceptos de discapacidad y salud en que se basan $^{5}$.

La esperanza de vida libre de discapacidad (EVLD) es un indicador muy útil para su uso poblacional porque el método de cálculo es sencillo y los resultados fáciles de interpretar. Se basa en datos de mortalidad ponderada en función de la prevalencia de discapacidad ${ }^{2,3}$ y tiene la gran venta- ja de que se ha hecho un importante esfuerzo para la estandarización de los conceptos, las fuentes de información y los métodos de cálculo gracias al trabajo de varios años de una red de investigación ${ }^{6}$. Además, la EVLD es uno de los indicadores recomendados por la Organización Mundial de la Salud para el seguimiento de las estrategias de salud para todos ${ }^{7}$, se ha incorporado como uno de los indicadores estructurales de la Unión Europea ${ }^{8}$ y están disponibles diversos cálculos para los países miembros ${ }^{9}$.

La mejora del estado de salud de la población es el principal objetivo y el mayor reto del sistema sanitario, motivo por el que es necesario buscar y mejorar indicadores fiables que permitan su monitorización. Desde que en España se ha producido la transferencia de la gestión de los servicios de salud a los gobiernos autonómicos adquiere una especial significación la disposición de estos indicadores desagregados geográficamente.

El objetivo del trabajo que se presenta es analizar la esperanza de vida libre de discapacidad al nacer para el año 1999 en España, según su distribución por comunidades autónomas (CCAA) y por sexo y compararla con la distribución geográfica del mismo indicador en el año 1986.

\section{MATERIAL Y MÉTODOS}

Datos: Los datos de mortalidad de España por CCAA de residencia de 1999 y los datos de población correspondientes a las proyecciones de población del mismo año proceden del Instituto Nacional de Estadística (INE) ${ }^{10}$. La información sobre discapacidad por edad, sexo y CCAA procede de la Encuesta sobre Discapacidades, Deficiencias y Estado de Salud (EDDES) de 1999 del INE ${ }^{11}$. Esta encuesta considera discapacidad "toda limita- 
ción grave que se padezca en la actualidad, que afecte de forma duradera a la actividad del que la padece (superior a un año) y que tenga su origen en una deficiencia". Incluye una lista de 36 ítems que se compone de limitaciones sensoriales, cognitivas, de percepción y de movilidad así como de discapacidades para las actividades de la vida diaria. En los menores de 6 años se recogen las posibles limitaciones futuras como consecuencia del estado de salud actual.

Los datos de la EVLD para 1986 por comunidades autónomas utilizados para comparar proceden del Ministerio de Sanidad y Consumo ${ }^{12}$. Los valores de la EVLD por CCAA calculados para 1986 y 1999 no son comparables porque los cuestionarios de la EDDES en que se basan son distintos, sin embargo sí que se pueden comparar las CCAA entre sí para el mismo año.

Métodos: Para el cálculo de la prevalencia de discapacidad por grupo de edad y sexo se consideró el número de personas que presentaban al menos alguna de las discapacidades de la lista, dividido por la población del grupo de edad correspondiente, para cada sexo y comunidad autónoma.

La EVLD se ha calculado mediante el método de Sullivan ${ }^{13}$. Para ello se construyeron las tablas de vida abreviadas para los hombres y las mujeres por CCAA y España del año 1999. Este método pondera el número de años que ha vivido la cohorte teórica de la tabla de vida, por la prevalencia de discapacidad (multiplicando por 1- la proporción de discapacitados) obteniéndose dos sub-cohortes: los años vividos con y sin discapacidad. Finalmente se obtiene la EV y la EVLD dividiendo el tiempo vivido (con discapacidad y sin discapacidad) por el número de supervivientes, en cada edad. Para el cálculo se emplearon grupos de edad quinquenales hasta los 85 años y más. Para conocer el grado de variabilidad de la EVLD se calculó el error estándar (EE) y el intervalo de confianza al 95\% (IC), según el método propuesto por el grupo REVES ${ }^{6}$. También se calculó la expectativa de discapacidad (D) (número medio de años que se espera sean vividos con discapacidad), restando la EVLD de la EV (EV-EVLD) y el porcentaje de esperanza de vida vivido sin discapacidades dividiendo la EVLD por la EV y multiplicando por cien [(EVLD/EV)*100].

La EVLD se interpreta como el número medio de años sin discapacidad que podría esperar vivir una persona en cada una de las diferentes edades cumplidas, sí se mantuviesen estables la tasa de mortalidad y la prevalencia de discapacidad por edad y sexo. Por su parte el porcentaje de esperanza de vida vivido sin discapacidades se considera una medida relativa del estado de salud o de calidad de vida.

\section{RESULTADOS}

En las tablas 1 y 2 se muestran la EV, la EVLD, los intervalos de confianza de ésta, la proporción que la EVLD representa sobre el total de años de EV, así como la expectativa de discapacidad para cada CCAA y por sexo. En ella se observa que en España en 1999 la esperanza de vida al nacer fue de 75,3 años para los hombres y de 82,3 para las mujeres y la EVLD de 68,5 y de 72,2 años respectivamente. En cambio, la proporción vivida libre de discapacidad era superior en los hombres $(91 \%)$ que en las mujeres $(87,7 \%)$ y la expectativa de discapacidad fue inferior para los hombres (6,8 años) que para las mujeres (10,1 años). La diferencia máxima entre los dos sexos se observó en Asturias para los dos indicadores con 6,7 años en la EVLD y 8,2 años en la EV.

Los datos muestran que las diferencias entre CCAA son mucho más acusadas 
Tabla 1

Esperanza de vida (EV) y Esperanza de vida libre de discapacidad (EVLD) por comunidades autónomas, Hombres. España, 1999

\begin{tabular}{|c|c|c|c|c|c|c|}
\hline CCAA & EV & EVLD & $\%$ & ED & $\mathbf{L I}$ & LS \\
\hline Andalucía & 73,99 & 66,07 & 89,30 & 7,92 & 65,75 & 66,38 \\
\hline Aragón & 76,09 & 70,19 & 92,25 & 5,90 & 69,62 & 70,75 \\
\hline Asturias & 73,94 & 67,06 & 90,70 & 6,88 & 66,313 & 67,81 \\
\hline Baleares & 74,46 & 68,16 & 91,54 & 6,30 & 67,32 & 69,01 \\
\hline Canarias & 74,19 & 68,48 & 92,30 & 5,71 & 67,88 & 69,08 \\
\hline Cantabria & 75,26 & 68,58 & 91,12 & 6,68 & 67,67 & 69,48 \\
\hline Castilla-La Mancha & 76,68 & 69,38 & 90,48 & 7,30 & 68,89 & 69,88 \\
\hline Castilla-León & 76,69 & 68,69 & 89,57 & 8,00 & 68,31 & 69,07 \\
\hline Cataluña & 75,57 & 68,64 & 90,83 & 6,93 & 68,26 & 69,06 \\
\hline Extremadura & 75,28 & 68,45 & 90,93 & 6,83 & 67,78 & 69,13 \\
\hline Galicia & 75,13 & 67,89 & 90,36 & 7,24 & 67,45 & 68,33 \\
\hline Madrid & 76,47 & 71,07 & 92,94 & 5,40 & 70,56 & 71,60 \\
\hline Murcia & 74,46 & 66,06 & 88,72 & 8,40 & 65,27 & 66,84 \\
\hline Navarra & 76,33 & 69,95 & 91,64 & 6,38 & 69,04 & 70,85 \\
\hline País Vasco & 75,31 & 70,14 & 93,14 & 5,17 & 69,61 & 70,66 \\
\hline Rioja & 75,92 & 71,66 & 94,39 & 4,26 & 70,77 & 72,55 \\
\hline Valencia & 74,65 & 68,81 & 92,18 & 5,84 & 68,37 & 69,26 \\
\hline Ceuta y Melilla & 74,56 & 67,87 & 91,03 & 6,69 & 66,50 & 69,13 \\
\hline España & 75,29 & 68,52 & 91,01 & 6,77 & 68,39 & 68,66 \\
\hline
\end{tabular}

$\%=(\mathrm{EVLD} / \mathrm{EV})^{*} 100$

$\mathrm{ED}=$ expectativa de discapacidad; $\mathrm{LI}$ = límite inferior del intervalo de confianza de la EVLD; LS = límite superior del intervalo de confianza de la EVLD.

Tabla 2

Esperanza de vida (EV) y Esperanza de vida libre de discapacidad (EVLD) por comunidades autónomas, Mujeres. España, 1999

\begin{tabular}{|c|c|c|c|c|c|c|}
\hline CCAA & EV & EVLD & $\%$ & ED & LI & LS \\
\hline Andalucía & 80,98 & 68,97 & 85,17 & 12,01 & 68,61 & 69,33 \\
\hline Aragón & 82,68 & 74,57 & 90,19 & 8,11 & 73,90 & 75,23 \\
\hline Asturias & 82,13 & 73,79 & 89,85 & 8,34 & 72,98 & 74,59 \\
\hline Baleares & 81,46 & 73,54 & 90,28 & 7,92 & 72,63 & 74,45 \\
\hline Canarias & 81,19 & 72,27 & 89,01 & 8,92 & 71,51 & 73,03 \\
\hline Cantabria & 83,13 & 73,19 & 88,04 & 9,94 & 72,13 & 74,25 \\
\hline Castilla-La Mancha & 82,41 & 71,95 & 87,31 & 10,46 & 71,37 & 72,53 \\
\hline Castilla y León & 83,87 & 72,07 & 85,93 & 11,80 & 71,64 & 72,50 \\
\hline Cataluña & 82,57 & 72,01 & 87,21 & 10,56 & 71,55 & 72,47 \\
\hline Extremadura & 82,16 & 71,39 & 86,89 & 10,77 & 70,56 & 72,22 \\
\hline Galicia & 82,54 & 72,35 & 87,65 & 10,19 & 71,87 & 72,83 \\
\hline Madrid & 83,79 & 74,71 & 89,16 & 9,08 & 74,07 & 75,35 \\
\hline Murcia & 80,87 & 68,82 & 85,10 & 12,05 & 67,95 & 69,70 \\
\hline Navarra & 83,65 & 74,96 & 89,61 & 8,69 & 73,93 & 75,99 \\
\hline País Vasco & 82,72 & 74,25 & 89,76 & 8,47 & 73,58 & 74,93 \\
\hline Rioja & 82,40 & 76,73 & 93,12 & 5,67 & 75,63 & 77,83 \\
\hline Valencia & 81,41 & 72,01 & 88,45 & 9,40 & 71,56 & 72,64 \\
\hline Ceuta y Melilla & 80,71 & 67,73 & 83,47 & 13,34 & 65,96 & 68,78 \\
\hline España & 82,31 & 72,21 & 87,73 & 10,10 & 72,07 & 72,36 \\
\hline
\end{tabular}

$\%=(\mathrm{EVLD} / \mathrm{EV}) * 100$

$\mathrm{ED}=$ expectativa de discapacidad; LI = límite inferior del intervalo de confianza de la EVLD; LS = límite superior del intervalo de confianza de la EVLD. 


\section{Figura 1}

Esperanza de vida y esperanza de vida libre de discapacidad por sexo y comunidades autónomas. España, 1999

\section{HOMBRES}

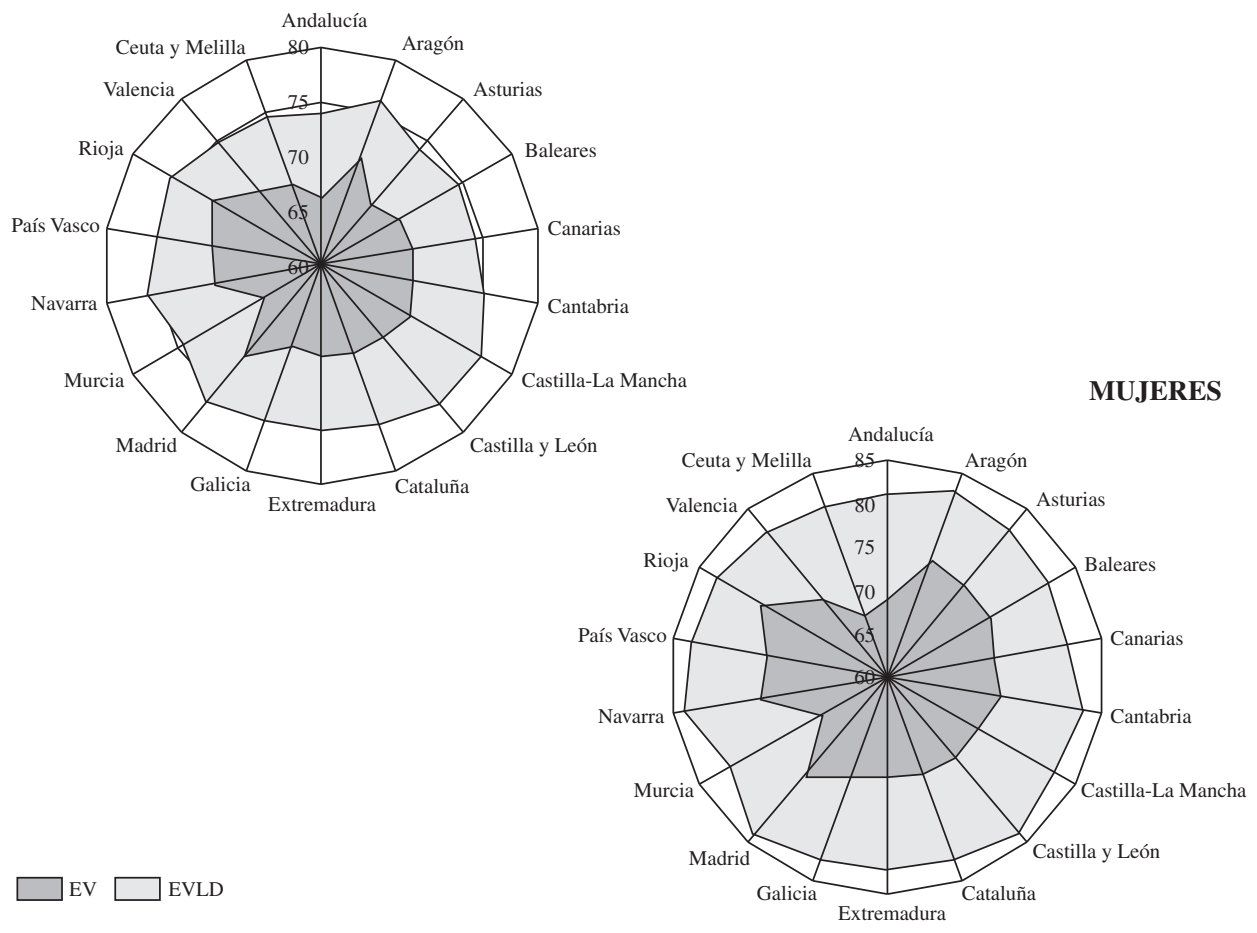

Figura 2

Esperanza de vida (EV) por sexo y comunidades autónomas. España, 1999

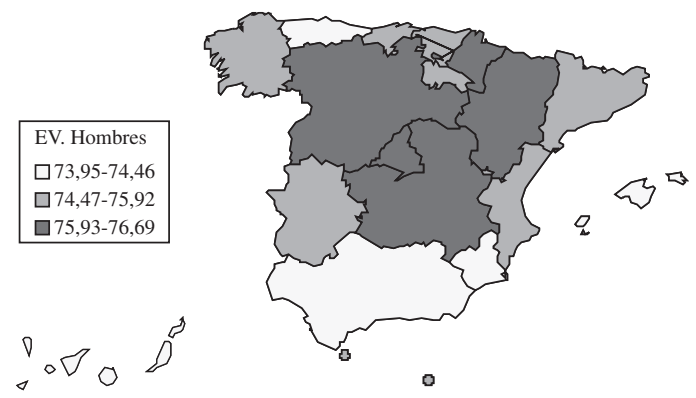


Figura 3

Esperanza de vida libre de discapacidad (EVLD) por sexo y comunidades autónomas. España, 1999

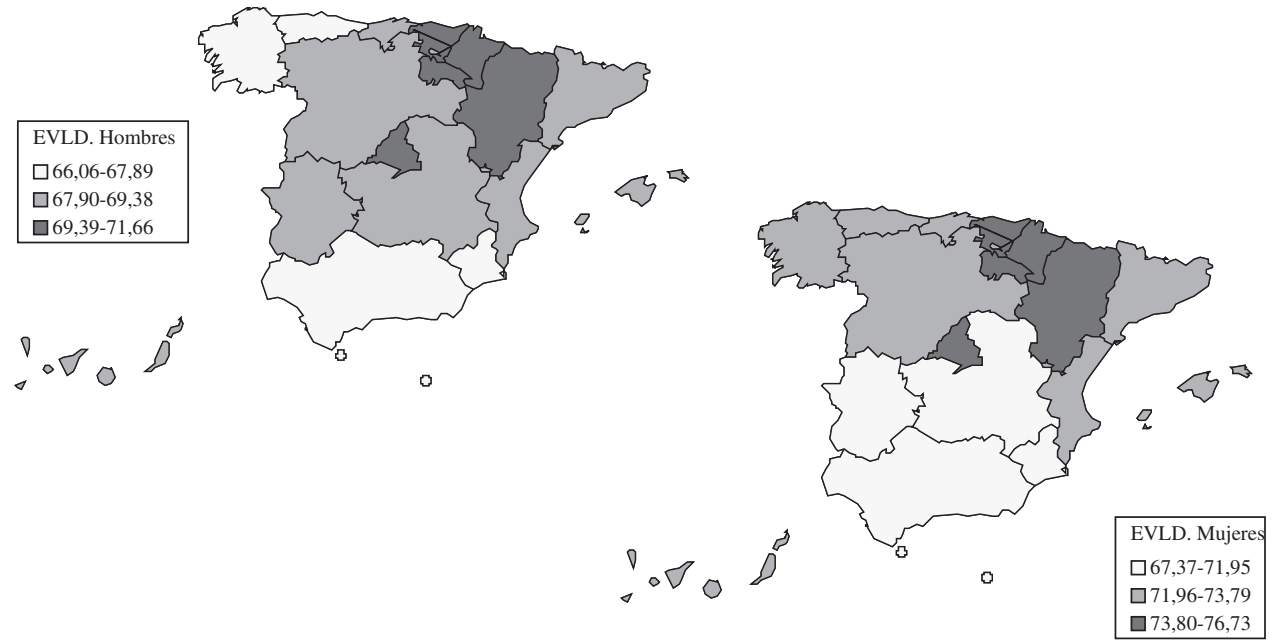

para la EVLD que para la EV. Así, la diferencia entre la comunidad con EV más alta y la más baja es de 2,7 años en hombres y de 3,2 años en las mujeres y de 5,6 y de 9,4 años respectivamente para la EVLD. Los hombres tienen una EVLD significativamente superior a la del conjunto español en seis comunidades (Aragón, Navarra, País Vasco, Rioja, CastillaLa Mancha y Madrid), significativamente menor en cuatro CCAA (Andalucía, Murcia, Galicia y Asturias) y no presenta diferencias significativas en el resto de CCAA. En cuanto a las mujeres siete CCAA están significativamente por encima de la media (Asturias, País Vasco, Navarra, Rioja, Aragón, Madrid y Baleares), 2 CCAA (Andalucía y Murcia) y las 2 ciudades autónomas están significativamente por debajo y el resto no muestra diferencias significativas.

La variabilidad geográfica se aprecia más claramente en los gráficos radiales (figura 1) que presentan la EV (la superficie total de la figura) y la EVLD al nacer (la superficie interior más oscura) para cada sexo por CCAA. Para magnificar las diferencias esta figura presenta los resultados a partir del valor 60 años que es el mínimo común para ambos indicadores en todas las CCAA. La irregularidad del contorno de las superficies coloreadas muestra las diferencias entre CCAA para cada indicador. Se observa que estas diferencias son mayores para la EVLD que para la EV y también que las CCAA con mayor EV no tienen siempre la mayor proporción de tiempo vivido libre de discapacidad. Los mapas presentan la distribución de los valores en tres rangos homogéneos y caracterizan un patrón geográfico algo diferente para la EV (figura 2) que para la EVLD (figura 3). La situación de la EV es más favorable en una amplia zona central de la península en los hombres y en el centro-norte peninsular en el caso de las mujeres. Para la EVLD, las zonas con mejor y peor salud se sitúan respectivamente más claramente al norte y al sur de la península.

En la figura 4 se presenta la posición de cada CA respecto del conjunto español de acuerdo al valor de la EVLD para los años 


\section{Figura 4}

Posición relativa de las comunidades autónomas según la Esperanza de vida libre de discapacidad en 1986 y 1999

\section{HOMBRES}

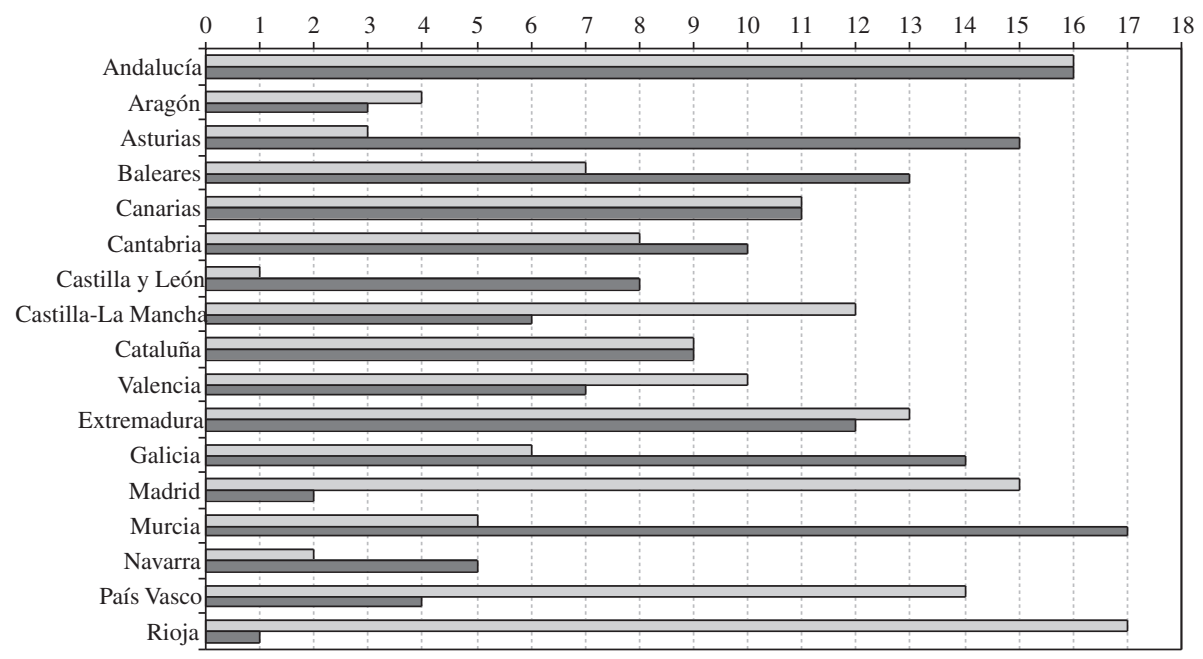

MUJERES

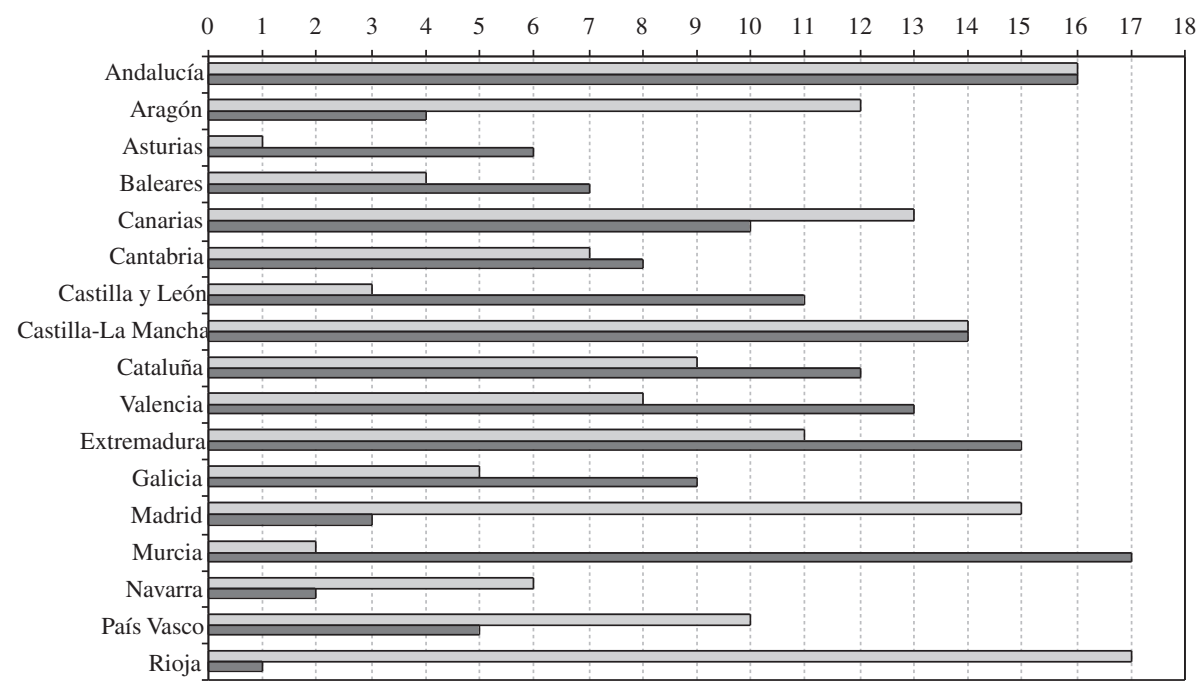


1986 y 1999 y para cada sexo, permitiendo apreciar el cambio sustancial de posiciones para algunas CCAA como Galicia, Asturias y Murcia que pierden su situación ventajosa por encima de la media española y de Madrid y la Rioja que la ganan.

\section{DISCUSIÓN}

En los resultados del estudio destacan diversos aspectos relevantes desde el punto de vista de la monitorización de la salud de la población española: la diversidad entre CCAA es más grande al considerar la EVLD que teniendo en cuenta solo la EV y que el patrón geográfico es distinto según se usa la EV o la EVLD. En general una mayor EVLD se corresponde con una mayor EV en términos absolutos, aunque esto no es siempre así (por ejemplo en Castilla-León), lo que significa que una EV más alta no expresa siempre un mayor nivel de salud. Esto se muestra claramente al analizar la EVLD en términos relativos ya que algunas CCAA presentan una proporción menor del tiempo vivido libre de discapacidad que otras (o lo que es lo mismo, viven más años pero pasan más tiempo con discapacidad, es decir con mala salud). Éste es en definitiva un ejemplo de la utilidad de este indicador para monitorizar la salud de la población.

La distribución de la EVLD por CCAA se ha presentado previamente, aunque de forma gráfica y agregada por rangos de valores $^{11}$, y en estimaciones para años distintos $^{12,14}$. Además de los valores numéricos del indicador el presente análisis ha incorporado el cálculo del error estándar de la EVLD, lo que permite hacer comparaciones entre CCAA y destacar aquéllas cuya diferencia por encima o por debajo del valor medio del conjunto español es estadísticamente significativa.

La disparidad entre CCAA es importante, con una diferencia de alrededor de tres años entre la comunidad con la EV más alta y la más baja. Este rango aumenta mucho al tener en cuenta la discapacidad de manera que la variación en la EVLD casi se duplica en los hombres y es tres veces mayor en las mujeres, poniendo de manifiesto una mayor desigualdad relacionada con la salud en este colectivo. Por otra parte, a pesar que la EV es más alta en las mujeres que en los hombres, la situación de salud en términos relativos de las mujeres es peor, de forma que viven más años que los hombres con altos niveles de discapacidad. Esta situación es consistente con la mayoría de estudios poblacionales que muestran sistemáticamente diferencias en el estado de salud entre los dos sexos y que también ha sido puesta de manifiesto en los estudios sobre la EVLD ${ }^{15}$, aunque en algunos casos parece que la diferencia entre los dos sexos se esta reduciendo ${ }^{16}$.

En lo que se refiere a las diferencias geográficas de EVLD entre CCAA, el patrón observado es consistente con el que se desprende del análisis de otros indicadores de mortalidad en que la zona sur de la península presenta una situación peor que el conjunto español ${ }^{14,17}$. Sin embargo este patrón es distinto al esperado en otras CCAA, donde a pesar de tener una EV elevada o media tienen una EVLD por debajo de la media española y aquellas CCAA con alta EV pero también con una alta proporción de años vividos con discapacidad, diferencias que no se habían puesto de manifiesto anteriormente.

Los cambios acaecidos en la posición de las CCAA entre 1986 y 1999 con respecto a este indicador de salud son también interesantes de destacar. Aunque los valores numéricos del indicador EVLD no son comparables entre los años 1986 y 1999, la distribución geográfica por CCAA y su posición respecto al conjunto español sí lo son para cada uno de los años. En el pasado, el patrón de "mejor salud" que la media española se concen- 
traba más en el noroeste y levante español. Mientras que en los datos de 1999 parece haberse desplazado a la zona nor-central de la península, con un cambio importante de posición respecto al conjunto para algunas CCAA.

Entre las razones para explicar las diferencias y los cambios observados destacan el desempeño de los servicios de salud ${ }^{18}$ y las condiciones socioeconómicas de las CCAA, aunque no siempre una mejora de estas condiciones implica la de la salud ${ }^{19}$. Estudios previos muestran que el indicador EVLD es sensible para detectar diferencias en el estado de salud de distintos grupos de población ${ }^{16,20}$ y que la variabilidad geográfica de los principales indicadores de salud se asocia con la distribución de variables socioeconómicas ${ }^{21}$, factor que podría explicar al menos en parte las diferencias observadas en este estudio tal como han mostrado otros trabajos con este indicador ${ }^{21,-23}$.

Tampoco hay que descartar el efecto de la medida de la discapacidad. Como en todas las encuestas, la EDDES de 1999 mide la discapacidad tal como es percibida por las personas entrevistadas y ésta es una de las razones mencionadas en la literatura para justificar posibles diferencias entre zonas con distinta cultura $^{24}$ incluso entre los dos sexos ${ }^{16}$. Así mismo, hay que tener en cuenta que la encuesta está referida a población general no institucionalizada, de manera que una oferta de plazas residenciales para ancianos y discapacitados distinta entre CCAA podría tener repercusión en las diferencias estimadas ${ }^{25}$. Este punto debería ser estudiado con más profundidad, puesto que los datos disponibles en la actualidad no permiten una respuesta fiable a esta hipótesis.

La comparación de la situación en España con la de otros países o regiones adolece del mismo problema que el encon- trado para comparar los indicadores elaborados con las encuestas de 1986 y 1999: la medida de la discapacidad. El esquema conceptual de la discapacidad ha evolucionado en los últimos años y con él las herramientas y los instrumentos para su formalización, tal como pone en evidencia el cambio en la clasificación internacional de discapacidades $^{26}$. Como consecuencia, existe una gran profusión de instrumentos distintos para medir la discapacidad en los diferentes estudios de población general (encuestas, paneles, etc.), que inevitablemente producen estimaciones diversas ${ }^{3}$. Así, aunque existen varios trabajos analizando la distribución y la evolución de la EVLD en los países europeos ${ }^{9,15}$ e incluso en España ${ }^{12,27}$, puesto que las medidas de discapacidad usadas son distintas, los datos no son comparables con los presentados aquí. Sin embargo, los principales resultados de este trabajo son consistentes con los que se presentan en la literatura científica en lo relativo a las diferencias entre los dos $\operatorname{sexos}^{15}$, a la capacidad de este indicador para detectar cambios ${ }^{16}$ temporales y a que la variabilidad geográfica es importante $^{21-23}$.

Finalmente, cabe señalar un concepto clave que se desprende del presente trabajo: una mayor EV no significa necesariamente un mayor nivel de salud, tal como se venía asumiendo tradicionalmente en el pasado. Por ese motivo los indicadores de esperanza de vida libre de incapacidad son un buen complemento a los indicadores clásicos de mortalidad para monitorizar la salud de la población española, de forma que sería necesario poder disponer de series de datos de discapacidad comparables que permitieran el cálculo continuado de este indicador a ese nivel territorial.

\section{AGRADECIMIENTOS}

El presente trabajo ha contado con ayuda parcial del ISC III (proyecto FIS PI052650). 


\section{BIBLIOGRAFÍA}

1. Word Health Organization. The World Health Report 2002: Reducing Risks, Promoting Healthy life. [consultada 12/07/2006]: Disponible en http://www.who.int/whr/2002/en/whr02_en.pdf

2. Gutiérrez Fisac JL, Regidor Poyatos E. Esperanza de vida libre de discapacidad: un indicador del estado de salud global. Med Clin (Barc). 1991 Mar 30; 96 (12):453-5.

3. Gispert R, Gutierrez-Fisac JL. Esperanza de vida saludable: pasado y presente de un indicador con futuro. Revisiones en Salud Pública. 1997; 5: 7-32.

4. Murray CJL. Quantifying the burden of disease: the technical basis for disability-adjusted life years. Bull World Health Organ. 1994; 72: 429-45.

5. Robine JM, Romieu I, Cambois E. Health expectancy indicators. Bull World Health Organ. 1999; 77 (2):181-185.

6. REVES : Réseau Espérance de Vie en Santé. International network on health expectancy and the disablement process. [consultada en Junio 2006]: Disponoble en: http://www.reves.net

7. World Health Organization. Regional Office for Europe. Health 21. The health for all policy framework for the WHO European Region. Copenhaguen: European Health for All Series 6; 1996.

8. Structural indicators. Eurostat. European Comision. [consultado Mayo 2006]: Disponible en: http://europa.eu/comm/eurostat/structuralindicators.

9. Robine JM, Jagger C, Clavel A, Romieu I. Disability- Free Life Expectancy (DFLE) in EU countries from 1991 to 2003. European health expectancy monitoring unit (EHEMU) Technical Report 1. 2004. [consultado en Junio 2006]: Disponible en: http://ec.europa.eu/health/ph_projects/2003/action1/docs/2003_1_08_rep1_en.pdf

10. INE. Instituto Nacional de Estadística. INEBASE. [consultada en julio 2005]: Disponible en: http://www.ine.es/inebase/index.html.

11. Instituto Nacional de Estadísticas. Encuesta sobre discapacidades, Deficiencias y Estado de Salud. Madrid 1999. Madrid 1999: Instituto Nacional de Estadística: 200. [consultado en Julio 2006]: Disponible en: http://www.ine.es/ineba$\mathrm{se} / \mathrm{cgi} / \mathrm{um} ? \mathrm{M}=\% 2 \mathrm{Ft} 15 \% 2 \mathrm{Fp} 418 \& \mathrm{O}=$ inebase $\& \mathrm{~N}$ $=\& \mathrm{~L}=$.
12. Regidor E, Rodríguez C, Gutiérrez Fisac JL. Indicadores de Salud. Tercera evaluación en España del programa regional europeo Salud para Todos. Madrid: Ministerio de Sanidad y Consumo; 1996.

13. Sullivan D F. A single index of mortality and morbidity. HSMHA Health Reports, 1971; 86:347-54.

14. Ministerio de Sanidad y Consumo. La salud de la población española en el contexto europeo y del sistema nacional de salud. Indicadores de salud. 2005. [consultada en Mayo 2006]: Disponible en: http://www.msc.es/estadEstudios/estadisticas/inforRecopilaciones/tabla/Indicadores5.pdf.

15. Perenboom RJM, Van Oyen H, Mustafova M. Health expectancies in European countries. In: Robine JM, Jagger C, Mathers CD, Crimmins EM, Suzman R (eds.) Determining health expectancies. Chichester: John Wiley and Sons Ltd; 2003. p. 359-76.

16. Perenboom RJM, Van Herten LM, Boshuizen HC, Van den Boss GAM. Life expectancy without chronic morbidity: trends in gender and socio-economic disparities. Pub Health Rep. 2005; 120 (1): 46-54.

17. Ruiz-Ramos M, Viciana-Fernandez F. Desigualdades en longevidad y calidad de vida entre Andalucía y España. Gac Sanit. 2004 Jul-Aug; 18(4): 260-7.

18. Gispert R, Torné MM, Barés MA. La efectividad del sistema sanitario en España. Gac Sanit. 2006; 20 (Supl 1): 117-26.

19. Regidor E, Ronda E, Pascual C, Martinez D, Calle ME, Dominguez V. Decreasing socioeconomic inequalities and increasing health inequalities in Spain: a case study. Am J Public Health. 2006 Jan; 96 (1): 102-8.

20. Martinez-Sanchez E, Gutiérrez-Fisac JL, Gispert R, Regidor E. Educational differences in health expectancy in Madrid and Barcelona. Health Policy $2001 ; 55$ : 227-231

21. Gutiérrez-Fisac JL, Gispert R, Solà J. Factors explaining the geographical differences in Disability Free Life Expectany in Spain. J Epidemiol Community Health 2000; 54: 451-455.

22. Mayer F, Ross N, Berthelot JM, Wilkins R. Disability-free life expectancy by health region. Health Rep. 2002; 13(4): 49-60

23. Bajekal M. Healthy life expectancy by area of deprivation: magnitude and trends in England, 1994-1999. Health Stat Q. 2005;25:18-27. 
24. Bullinger M. International comparability of health interview surveys: An overview of methods and approaches. In Nosikov A and Gudex C (eds.) EUROHIS: Developing common instruments for health surveys. WHO Biomedical and health research, vol57. Amsterdam: IOS Press; 2003.

25. Edad \& Vida. Estudio del modelo de atención a las personas mayores con dependencia en España. 2004: [consultado en febrero 2006]: Disponible en: http://www.edad-vida.org
26. Ayuso-Mateos JL, Nieto-Moreno M, SanchezMoreno J, Vazquez-Barquero JL. Clasificación Internacional del Funcionamiento, la Discapacidad y al Salud (CIF): aplicabilidad y utilidad en la práctica clínica. Med Clin (Barc). 2006 Apr 1; 126(12):461-6.

27. Gispert R, Puig X, Puigdefabregas A, Tresserras $R$, Busquets E. Esperanza de vida libre de incapacidad y esperanza de vida en buena salud en Cataluña 1994-2000. Med Clin (Barc). 2003; 121 Suppl 1:128-32. 
